\title{
The Okabayashi Radical Hysterectomy: Basic Principle and Step-by-Step Procedure
}

\author{
Ikuo Konishi, MD, $\mathrm{PhD}^{1,2}$ \\ ${ }^{1}$ Department of Gynecology and Obstetrics, Kyoto University \\ Graduate School of Medicine, Kyoto, Japan \\ 2 National Hospital Organization, Kyoto Medical Center, Kyoto, Japan
}

Surg J (NY) 2021;7(suppl S2):S57-S69.

\begin{abstract}
Address for correspondence Ikuo Konishi, MD, PhD, National Hospital Organization, Kyoto Medical Center, 1-1 Fukakusa Mukaihata-cho, Fushimi-ku, Kyoto 612-8555, Japan (e-mail: konishi@kuhp.kyoto-u.ac.jp).
\end{abstract}

\author{
Abstract \\ Keywords \\ - Okabayashi radical \\ hysterectomy \\ - basic principle \\ - step-by-step \\ procedure \\ - cervical cancer
}

Abdominal radical hysterectomy is the standard operation for stage IB-IIB cervical cancer in the world, which was established by Professor Hidekazu Okabayashi in 1921. In this article, the basic principle and step-by-step procedure of Okabayashi radical hysterectomy are presented, especially for young doctors to understand clearly the important points of this surgery and to realize its radicality and safety for better survival and quality of life of patients with cervical cancer.

\section{Beginning for Development of Radical Hysterectomy}

There has been a long history of the establishment of radical hysterectomy for cervical cancer. ${ }^{1}$ At the end of 19 th century, Dr. Ernst Wertheim (1864-1920) of Vienna tried to develop abdominal surgery for invasive cervical cancer, and performed the first operation for Madame Rudd in 1898. In 1906, he demonstrated the treatment results of more than 300 cases in Chicago, ${ }^{2}$ but his surgery was not accepted due to high incidence of operative morbidity and mortality in the era that saw the emergence of radiotherapy. In the United States, the Wertheim surgery for cervical cancer was revisited by Dr. Joe Vincent Meigs (1892-1963), Professor of Harvard Medical School, in $1944^{3}$ and has become popular since the 1950s.

On the other hand, in the same era as Wertheim, trials of surgical approach had been maintained by Dr. Wilhelm Latzko (1863-1945) in Europe and by Dr. Shohei Takayama (18621925), the 2nd Professor of Kyoto University, in Japan. Dr. Takayama is the originator of radical surgery in Japan, and demonstrated his surgery at the 16th Annual Meeting of Japan Society of Gynecology in 1917. Then, in 1921 Dr. Hidekazu
Okabayashi (1884-1953), the 3rd Professor of Kyoto University ( - Fig. 1), developed the Okabayashi method, improving delicately the Takayama operation. ${ }^{4}$ Thus, the ultimate standard of radical hysterectomy was established in the early 20th century in Japan. Since then, the original procedure of Okabayashi operation has enthusiastically been improved and modified by many gynecologic surgeons for safety and easier learning. Until now, however, the basic principle of his surgery has been maintained for realizing the radicality.

\section{Basic Principle of Okabayashi Radical Hysterectomy}

The most important point of Okabayashi method is the feasibility of radical resection of three ligaments supporting the uterine cervix, i.e., cardinal ligament, rectovaginal ligament, and vesicouterine ligament ( - Fig. 2). He clearly demonstrated how to explore the pelvic cavity and identify the three ligaments systematically for their resection at the appropriate point. ${ }^{5}$ To complete, it is necessary to explore the three retroperitoneal spaces, i.e., paravesical space, pararectal space (Latzko's space), and pararectal space (Okabayashi). Identification of each published online October 12, 2021
DOI https://doi.org/ $10.1055 / \mathrm{s}-0041-1736176$. ISSN 2378-5128.

\footnotetext{
(C) 2021. The Author(s).

This is an open access article published by Thieme under the terms of the Creative Commons Attribution-NonDerivative-NonCommercial-License, permitting copying and reproduction so long as the original work is given appropriate credit. Contents may not be used for commercial purposes, or adapted, remixed, transformed or built upon. (https://creativecommons.org/ licenses/by-nc-nd/4.0/)

Thieme Medical Publishers, Inc., 333 Seventh Avenue, 18th Floor New York, NY 10001, USA
} 


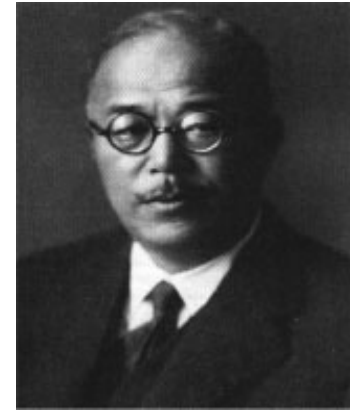

Fig. 1 Professor Hidekazu Okabayashi (1884-1953).

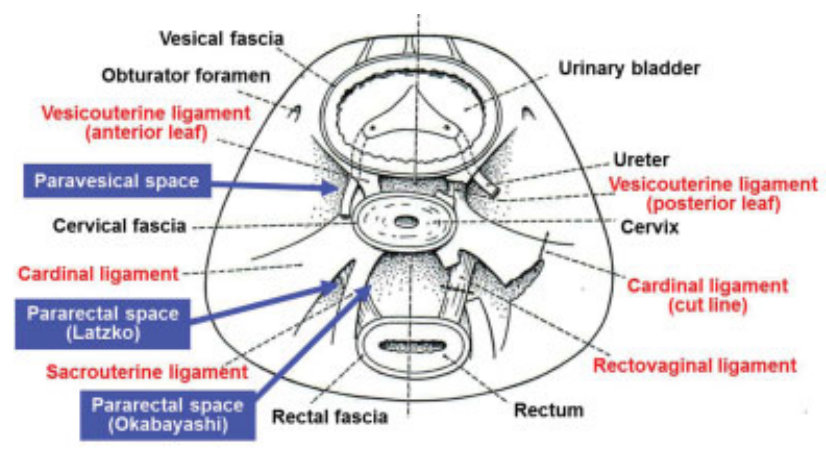

Fig. 2 Basic principle of Okabayashi radical hysterectomy. Radical resection of three ligaments supporting the uterine cervix, i.e., cardinal ligament, rectovaginal ligament, and vesicouterine ligament, is feasible after exploration of the three retroperitoneal spaces, i.e., paravesical space, pararectal space (Latzko's space), and pararectal space (Okabayashi). (Source: Ogura T. Radical hysterectomy: Okabayashi original procedure. In: Kobayashi T, et al. eds. Cervical Cancer. Tokyo: Nakayama Shoten; 1970.)

ligament enables us to extirpate cancer with free margins. Especially for the vesicouterine ligament, he discovered the two different planes surrounding the ureter, i.e., anterior and posterior leaves. After the exploration and cutting of the two leaves, the whole vagina with paracolpium appears, and its amputation becomes possible at the appropriate level being free from the vaginal invasion.

It is said that the original Okabayashi surgery streams always from posterior to anterior. ${ }^{6}$ First, the treatment of infundibulopelvic ligament, broad ligament, and then round ligament is done. The ureter is identified and uterine artery is cut. The treatment of the three ligaments starts from the sacrouterine ligament, and proceeds to the exploration of pararectal space (Okabayashi) and then paravesical space. After that, the rectovaginal and the cardinal ligaments are clamped, cut, and ligated (-Fig. 3A). Finally, the surgery moves to the treatment of anterior leaf of vesicouterine ligament, mobilization of ureter, and treatment of posterior leaf of vesicouterine ligament ( - Fig. 3B). After amputation of the vagina, pelvic lymphadenectomy is performed.

\section{Modification of Okabayashi Radical Hysterectomy}

The first modification of the Okabayashi original procedure was proposed by Dr. Kyusaku Ogino (1882-1975) from Tokyo
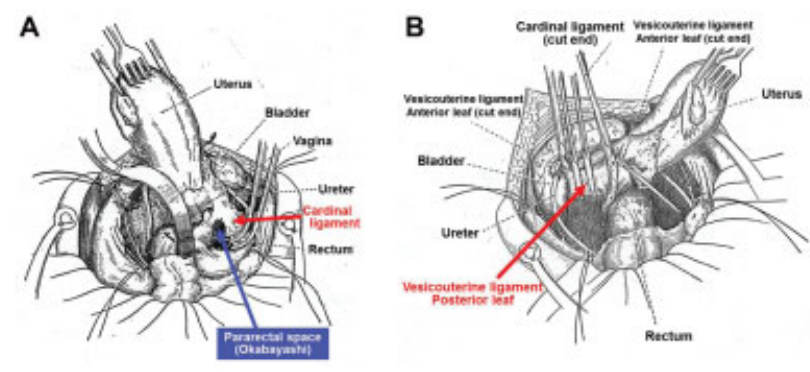

Fig. 3 Original procedure of Okabayashi radical hysterectomy. After exploration of pararectal and paravesical spaces, the rectovaginal and then the cardinal ligaments are clamped, cut, and ligated (A). Then, the surgery proceeds to the treatment of anterior leaf of vesicouterine ligament, mobilization of ureter, and posterior leaf of vesicouterine ligament (B). (Source: Okabayashi H. Radical Hysterectomy for Cervical Cancer. Tokyo: Nippon Isho Shuppan; 1952.)

University. After watching the surgery by Dr. Okabayashi, he advocated that lymphadenectomy might be performed before treatment of the three ligaments. Since then, most gynecologic surgeons have accepted his idea, and do pelvic lymphadenectomy first for clarification of pelvic anatomy and achievement of safety.

The most serious complication after radical hysterectomy is urinary dysfunction. Dr. Takashi Kobayashi (1909-1992), Professor of Tokyo University, developed the surgical procedure for sparing the autonomic nerves, ${ }^{7}$ which has recently been standardized by Dr. Shingo Fujii, the 8th Professor of Kyoto University. ${ }^{8}$

Locally advanced cervical cancer with severe parametrial involvement has not usually been indication of surgical treatment. However, Dr. Ryukichi Mibayashi (1898-1977), the 4th Professor of Kyoto University, developed "super-radical hysterectomy," in which the cardinal ligament is completely extirpated from the pelvic wall by co-resection of internal iliac artery and vein, even for stage IIIB patients. ${ }^{9}$ Currently, such extensive surgery is still useful for patients in whom the metastatic or recurrent tumors are present along the pelvic wall or on the iliopsoas fascia. ${ }^{10}$ The similar operation has independently been proposed by Dr. László Ungár ${ }^{11}$ and Dr. Michael Höckel, ${ }^{12}$ and is designated as laterally extended parametrectomy or laterally extended endopelvic resection.

I myself learned the procedure of radical hysterectomy from Dr. Toshio Nishimura, the 5th Professor of Kyoto University, and Dr. Masahiko Ukita at Kurashiki Central Hospital, and read the books written by Dr. Kobayashi at Tokyo University and by Dr. Toshiro Fujiwara at Tenri Hospital, and then improved the techniques for the safety of patients and the education for young doctors.

\section{Preoperative Evaluation}

Clinical conference before surgery is very important for the patient and the doctors. All the medical staffs, not only gynecologists but also radiologists and pathologists, if possible, should gather and discuss the extent of disease, the feasibility of surgery, and the actual procedure of operation, to achieve the radicality and safety of radical hysterectomy. ${ }^{1}$ 
Assessment of lymph node metastasis by computed tomography (CT) and magnetic resonance imaging (MRI) is essential for prognosis of patients. The range of lymphadenectomy will be extended to para-aortic area if needed. More important issue is the degree of local invasion of cancer to the parametria. Invasion of cancer into the rectovaginal or cardinal ligaments can be recognized by palpation and MRI, and is successfully treated by the standard or extended procedure of radical hysterectomy. However, the invasion into the vesicouterine ligaments surrounding the ureter cannot be recognized by palpation, and therefore, close observation of MR images is needed. If the invasion is present, the procedure of radical hysterectomy should be converted to surgery that includes the partial resection of ureter and bladder with their neoanastomosis. Thus, preoperative planning of the surgery is essential for the radical and safe operation for the patient.

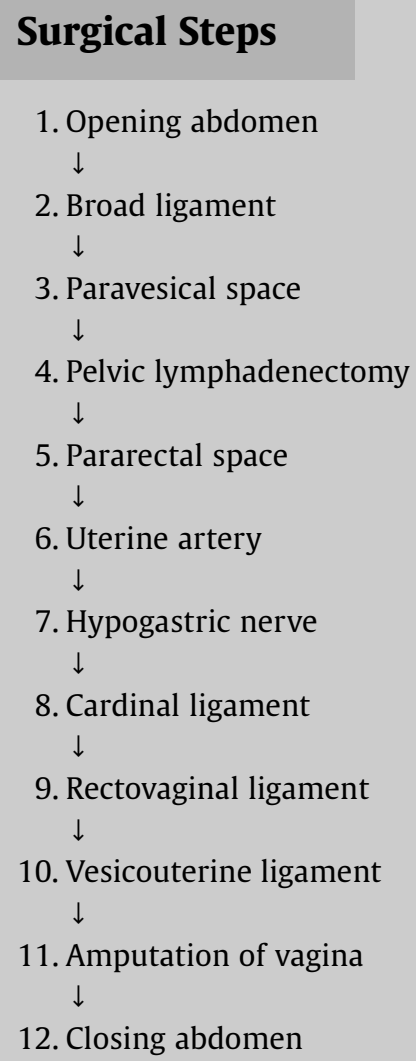

\section{Explanation of Step-by-Step Procedures}

It is generally said that the procedure of radical hysterectomy is complicated and relatively difficult to master. However, it will not be the case after reading this article carefully. Since I have fully explained how to perform the radical hysterectomy uneventfully, readers can master the actual procedure.

\section{Opening Abdomen}

Patient is laid in the supine position and urethral catheter is inserted for continuous bladder drainage. The operator incises the abdominal wall longitudinally from the pubis toward the umbilicus, and then extends the skin incision upward to approximately $3 \mathrm{~cm}$ above the umbilicus.

Before initiating the surgery, the operator should examine the pelvic organs, especially palpating the parametrium. Reassessment of parametrial invasion is very important to assess the feasibility of radical hysterectomy. Ascitic fluid is presented to pathology laboratory for cytological examination, which is prognostic indicator especially for cervical adenocarcinoma. If positive, partial omentectomy is usually performed to examine the microscopic peritoneal dissemination.

Throughout the surgical procedures, the uterus is always maintained in the appropriate traction by the assistant. Usually, a pair of long and straight Kocher clamps are placed between the uterus and the adnexa. Various retractors are also used to show the lymphadenectomy field and to open the three retroperitoneal spaces for the treatment of ligaments.

\section{Broad Ligament}

The first step is the same as simple hysterectomy, i.e., ligation and cutting of the round and infundibulopelvic ligaments, followed by incision of broad ligaments. If the patient is young and the cancer is squamous cell carcinoma at stage IB or less, the ovaries are usually preserved. In such case, the ovarian ligament is clamped, cut, and ligated, instead of infundibulopelvic ligament. Recently, salpingectomy is frequently performed considering the possible tubal origin of ovarian cancer. To do so, mesosalpinx is clamped, cut, and ligated several times. All of the above-mentioned procedures are done for both sides, and now, all of the peritoneal surfaces are incised, except the posterior leaf of broad ligament and the cul-de-sac peritoneum. From now, the surgical procedures in the retroperitoneal space start.

\section{Paravesical Space}

In the modern radical hysterectomy, pelvic lymphadenectomy precedes the treatment of the three ligaments. The important first step to prepare lymphadenectomy is the opening of paravesical space. To do so, the operator explores the space, using both index fingers, vertically from beneath the cut end of round ligament, medial to the external iliac artery, and can find easily the space that reaches to the pelvic floor ( - Fig. 4A). Connective tissue in this space is very loose, and the procedure can be done with no resistance. Importantly, this space should be lateral to the internal iliac artery (lateral umbilical ligament) ( Fig. 4B), because there are several arterial branches running medially from the internal iliac artery.

After opening, the operator will extend the paravesical space cranially and caudally, sliding the fingers along the external iliac vessels. Now, one can clearly recognize the running of the external iliac vein under the iliac artery ( - Fig. 4B). The index fingers also reach downward to the levator ani muscle of pelvic floor. Then, the retractor is inserted into the paravesical space, and its mild traction toward the pubic bone is maintained by the assistant. Exploration results in the formation of triangle-shaped space (-Fig.5), which is composed by the lateral (external iliac vessels), the medial (internal iliac artery), and the base (pubic bone). This triangle is the surgical field for pelvic lymphadenectomy. 


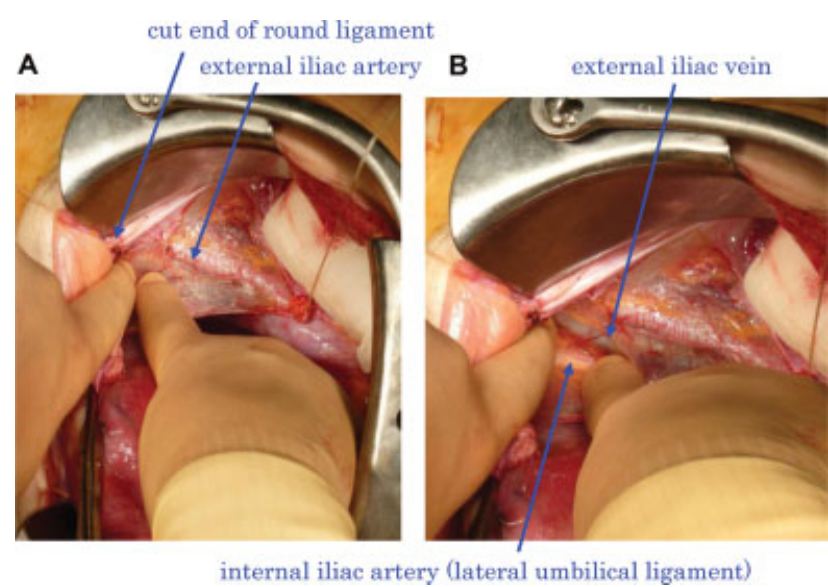

Fig. 4 Exploration of paravesical space. From beneath the cut end of round ligament, both index fingers are inserted vertically, downward into the space, medial to the external iliac vessels (A). This is paravesical space, which should be lateral to the internal iliac artery (lateral umbilical ligament) (B).

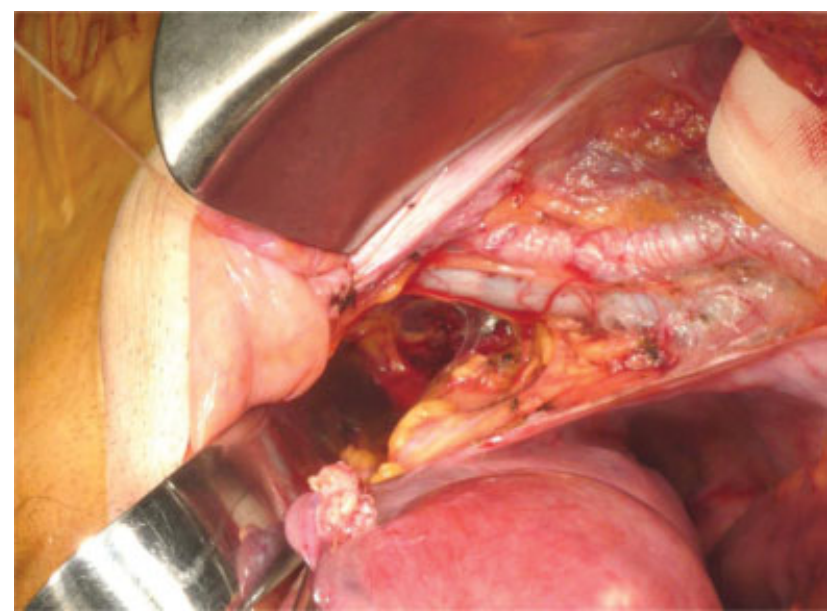

Fig. 5 Triangle of paravesical space. The paravesical space is extended caudally and cranially, and also reaches downward to the pelvic floor. Insertion of the retractor and its mild traction toward the pubis forms the triangle-shaped space. This is the surgical field of pelvic lymphadenectomy.

\section{Tips and Warnings}

The surgical procedure exploring the paravesical space is very important not only in radical hysterectomy but also in other gynecologic and urologic surgeries, especially when the anatomy of pelvic organs is complicated. The landmarks for opening the paravesical space are the cut end of round ligament and the external iliac artery. After exploring this space, the operator can find easily the ureter, the obturator nerve, and the uterine vessels. Thus, it becomes possible for the operator to continue the following surgical procedures without injuring the urinary tract or causing massive bleedings.

\section{Pelvic Lymphadenectomy}

Surgery for lymphadenectomy is not the extirpation of only lymph nodes but the systematic dissection of all lymphatic tissues. To do so, skeletonization of the blood vessels is an ideal approach to lymphadenectomy, because the lymphatic channels and nodes are present within the soft tissues on the surface of main blood vessels. After skeletonization, only the adventitia of the blood vessels remains. It is also advantageous to prevent unnecessary bleeding from the blood vessels. Risk of bleeding is much higher at skeletonization of big vein than that of big artery, and therefore, the surgical procedure should start from artery and then proceed to vein. For the procedure of dissection, we usually use the aspirator, which can remove the adipose and loose connective tissues, but not damage the lymphatic and blood vessels. By the aspirator action, the objective tissues including the lymph nodes become apparent. After clarifying the anatomy, we use the unipolar electric cautery, rather than the scissors, for the dissection. Bipolar electric cautery is also useful to manage the bleeding from small vessels.

Pelvic lymph vessels and nodes are classified as two distinct lines, i.e., lateral and medial chains. Lateral chain consists of lateral suprainguinal, external iliac, and superficial common iliac nodes. Medial chain consists of medial suprainguinal, obturator, internal iliac, and deep common iliac nodes. Dissection of lateral chain should precede that of medial chain.

\section{Lateral Chain}

The lymph nodes that should be dissected first are the external iliac nodes, because the risk of unnecessary trauma and unexpected bleeding is the least. To do so, the operator should skeletonize first the external iliac artery, find small nodes, and remove them (-Fig. 6).

The second are suprainguinal nodes. The assistant lifts up the operation side of self-retaining retractor and also attaches another retractor to the inguinal region of abdominal wall to maximize the operative field. The operator

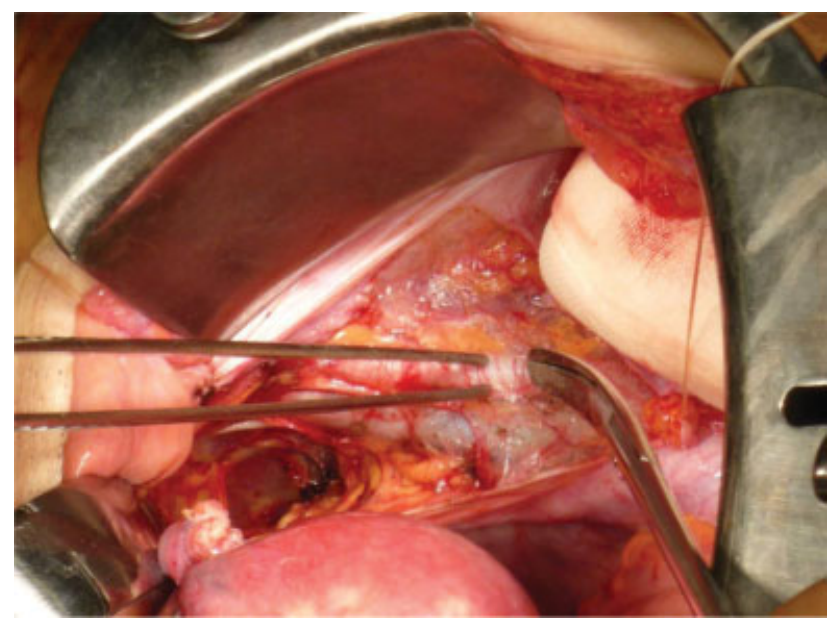

Fig. 6 Lymphadenectomy of lateral chain: external iliac nodes. During skeletonization of external iliac artery, external iliac nodes appear on the artery and are dissected. This is the first step of lymphadenectomy. 

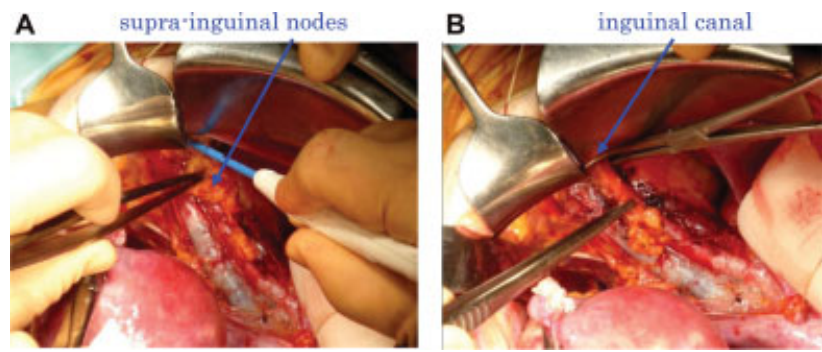

Fig. 7 Lymphadenectomy of lateral chain: lateral suprainguinal nodes. Removal of connective and adipose tissues on the fascia of iliopsoas muscle and on the external iliac vessels using aspirator and monopolar cautery discloses the presence suprainguinal nodes (A). Then, lymph channels going into the inguinal canal are clamped, cut, and ligated (B).

removes the adipose and loose connective tissues on the fascia of iliopsoas muscle using the aspirator and monopolar cautery, and gradually approaches the suprainguinal region ( - Fig. 7A). Genitofemoral nerve should be preserved. Be careful about a small vein running vertically on the surface of artery (circumflex iliac vein), which is usually preserved but is ligated if injured. During the dissection, large suprainguinal lymph nodes become evident (-Fig. 7A). The lymph channels going from the nodes into the inguinal canal are clamped, cut, and ligated (-Fig. 7B). Ligation of lymph vessels is important to prevent the formation of lymphocyst or lymphorrhea after the surgery.

The third are superficial common iliac nodes. The assistant should place the retractor on the common iliac artery and slide it upward carefully, and the ureter should be placed medial to the retractor (-Fig. 8). First, the operator removes the loose connective tissues on the common iliac vein that is present lateral and beneath the common iliac artery. Vasa vasorum on the artery is successfully managed with bipolar cautery. Then, the common iliac nodes on the artery and vein become apparent, and are removed. The lymph channels going upward from the nodes are clamped, cut, and ligated. Now, lymphadenectomy of the lateral chain is completed.

\section{Tips and Warnings}

Since the common iliac vein goes through under the artery and is hiding just lateral to the artery, the operator should identify the vein first to prevent its injury and massive bleeding. To do so, using the aspirator, the operator removes the connective tissue, which exists lateral to the common iliac artery, and finds the running of the vein. After clarification of the anatomy, the common iliac nodes on the artery and vein become apparent, and can be removed safely.

\section{Medial Chain}

Before lymphadenectomy of medial chain, it is important to identify the obturator nerve for clarifying the anatomy of

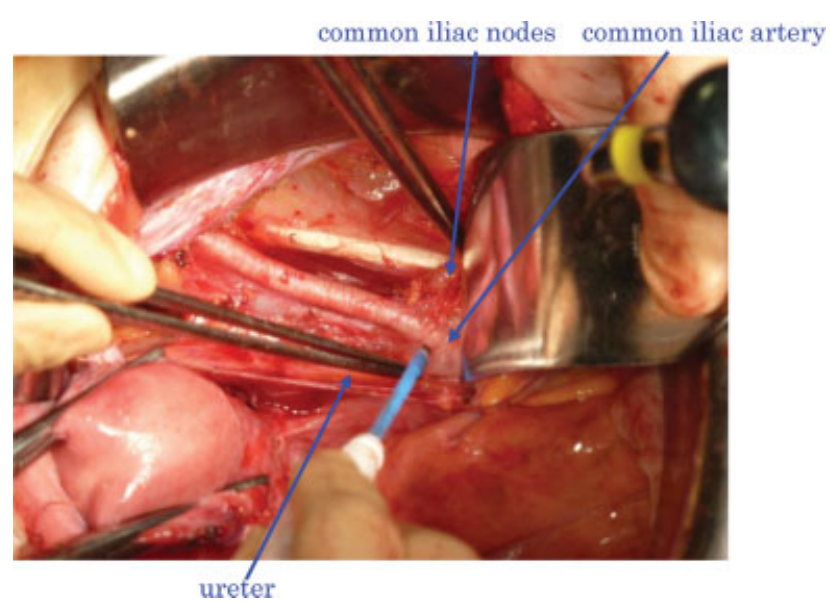

Fig. 8 Lymphadenectomy of lateral chain: superficial common iliac nodes. Application of retractor and its mild traction upward disclose the surface of common iliac artery, and push the ureter medially. First, the aspiration of connective tissues lateral to the artery is needed to identify the common iliac vein running through under the artery. After such clarification of anatomy, the common iliac nodes are dissected from those vessels.

obturator fossa. The assistant should lift up the external iliac artery and vein using the scissors or other small retractor, and the operator uses the aspirator to remove the loose connective tissues under the external iliac vessels (-Fig. 9A). Now, one can find the fascia of iliopsoas muscle near the lateral wall of pelvis. Then, the white-colored, thick string appears. This is the obturator nerve, which is now partially isolated (-Fig. 9B). It is important to recognize the height level of the running nerve, since the medial chain exists in the obturator fossa between the external iliac vein and the obturator nerve. The operator should not touch the obturator nerve directly by the monopolar cautery, because it will cause nerve stimulation and muscle contraction, which will shake the operative field. And, it is better not to touch the connective tissues under the obturator nerve, because the vascular-rich cardinal ligament is present here and will be explored later.

First, internal iliac nodes on the internal iliac artery and on the uterine artery should be dissected. These nodes are classically known as "interiliac nodes." It is important to dissect these nodes completely, since they harbor metastases frequently. The connective tissues containing the lymph
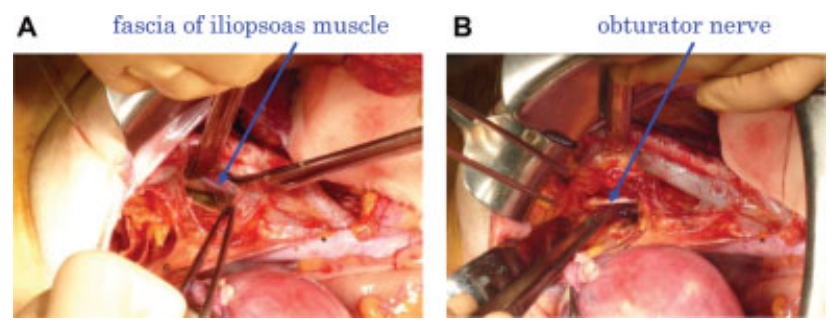

Fig. 9 Lymphadenectomy of medial chain: identification of obturator nerve. Before identifying the nerve, the external iliac vein is tentatively skeletonized, and the fascia of iliopsoas muscle becomes visible (A). During the removal of connective tissue under the iliac vein by aspirator, the white-colored string appears. This is the obturator nerve (B). 

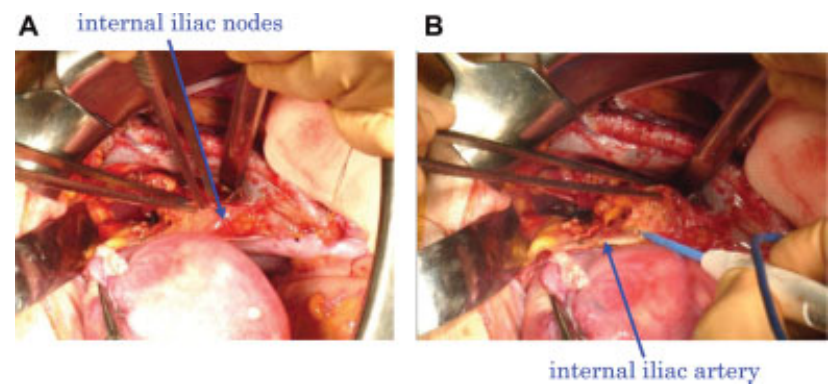

Fig. 10 Lymphadenectomy of medial chain: internal iliac nodes. First, internal iliac nodes on internal iliac artery and on the uterine artery are dissected. Isolation of the arteries discloses the lymphatic tissues continuous with them $(A)$, which are dissected using monopolar cautery (B).
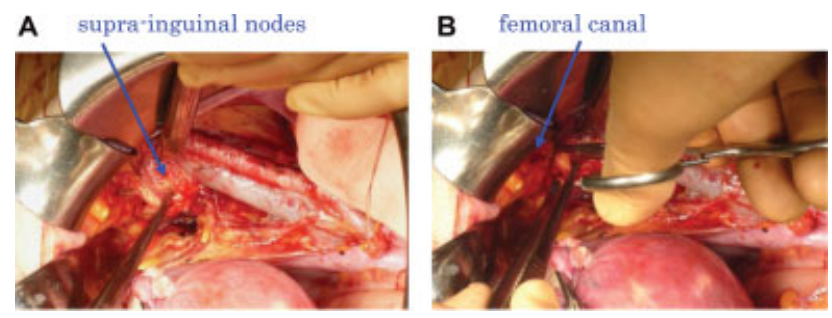

Fig. 11 Lymphadenectomy of medial chain: medial suprainguinal nodes. All of tissues containing lymph nodes between the external iliac vein and obturator nerve should be removed. Caudal skeletonization of the external iliac vein discloses the presence of suprainguinal nodes (A), and then lymph channels going into the femoral canal are clamped, cut, and ligated (B).

nodes cover superficially the obturator fossa, and so, isolation of the internal iliac artery discloses the lymphatic tissues continuous with them ( - Fig. 10A), which will be dissected using the monopolar cautery ( - Fig. 10B).

Now, it is time to dissect thoroughly the medial chain continuing from the suprainguinal nodes to the obturator nodes, which is located in the space between the external iliac vein and obturator nerve. Complete isolation of the obturator nerve is essential. Here, the operator can also find the obturator artery and vein, running parallel to the obturator nerve, which are usually preserved during the dissection. However, if these vessels will disturb the surgical procedure, they can be clamped, cut, and ligated.

Caudal skeletonization of the external iliac vein reveals gradually the presence of large medial suprainguinal nodes ( - Fig. 11A), which will be detached from the vessels and from the nerve by the aspirator and cautery. Finally, the lymphatic channels going into the femoral canal are identified, and are clamped, cut, and ligated (-Fig. 11B).

Next target is the obturator nodes. Since they are continuous with the medial suprainguinal nodes, both tissues can be dissected en bloc. Complete removal of the obturator nodes is very important because they harbor metastases most frequently. The lymphatic tissues are detached from the obturator nerve and small vessels coming from the pelvic wall ( - Fig. 12A). The lymphatic channels of medial chain going upward under the external iliac vein are identified, and so, they will be clamped, cut, and ligated here tentatively
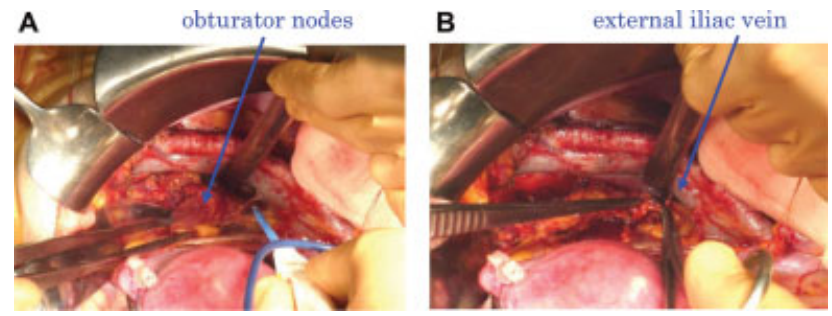

Fig. 12 Lymphadenectomy of medial chain: obturator nodes. Obturator nodes are continuous with the medial suprainguinal nodes. These lymphatic tissues are detached from the obturator nerve and small vessels of the pelvic wall (A). Lymph vessels going up under the external iliac vein are clamped, cut, and tentatively ligated (B). Cut end is marked.

(-Fig. 12B). Be careful not to injure the bifurcation point of the external and internal iliac veins from the common iliac vein. Also, be careful not to cut the obturator nerve here. The cut end of medial chain should be marked, because the continuous lymphatic tissues will be treated soon later in the different surgical field.

Now, most procedures of pelvic lymphadenectomy have been completed, and the triangle space clearly shows the anatomical relationship among the external and internal iliac vessels, the ureter, the obturator nerve, and pelvic side wall ( - Fig. 13).

\section{Tips and Warnings}

Before initiating the dissection of medial chain, the external iliac artery and vein are lifted up for identification of the obturator nerve. Here, it is important for the operator to watch the fascia of iliopsoas muscle lateral to the external iliac vein, and recognize the anatomical relationship among vessels, nerve, and pelvic wall. The metastatic or recurrent tumor is frequently present here and adheres to the fascia. In such case, the operator can

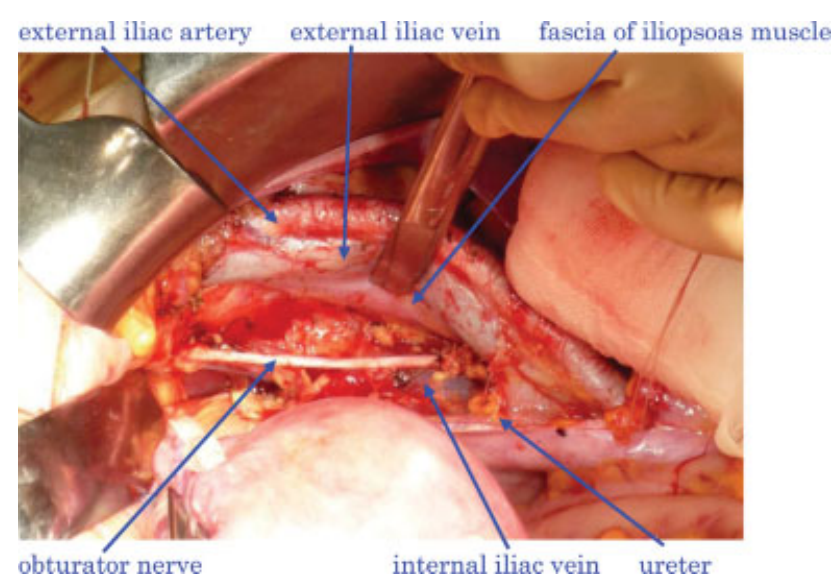

Fig. 13 Landscape of the triangle space after lymphadenectomy. Most procedures of pelvic lymphadenectomy have been completed, and now the operator can recognize again the running of external iliac artery, vein, fascia of iliopsoas muscle, pelvic wall, obturator nerve, internal iliac artery, and ureter. The main trunk of internal iliac vein is partially seen. The lymphatic tissues under the obturator nerve will be dissected later. 

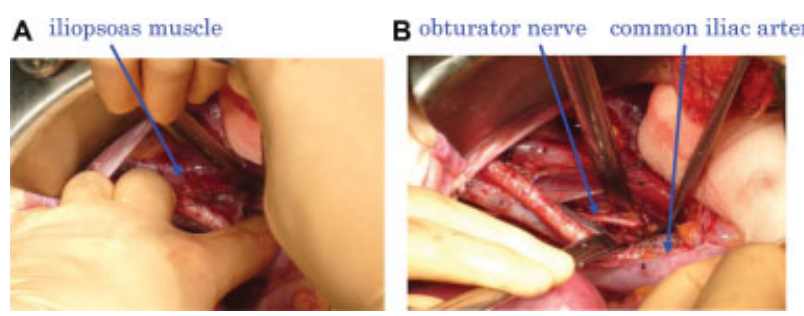

Fig. 14 Lymphadenectomy of deep common iliac nodes. The operator explores a different surgical field, inserting the scissors and the index finger between the common iliac artery and iliopsoas muscle (A). The obturator nerve and the marked cut end of lymph channels can be found. The deep common iliac nodes continuous with the obturator are dissected (B).

perform curable operation via complete removal of recurrent tumor with co-resection of the fascia. After resection of the fascia, red-colored striated muscle can be seen. Thus, the gynecologic surgeon should be familiar with the iliopsoas muscle of pelvic side wall.

\section{Deep Common Iliac Area}

Finally, the deep common iliac nodes should be dissected. To do so, the operator explores the upper space between the common iliac artery and the iliopsoas muscle, using fingers, scissors, and aspirator (-Fig. 14A). Then, one can find the obturator nerve, the common iliac vein, and the marked cut end of medial chain of lymphatics (-Fig. 14B). The superior gluteal vein is also seen here. The lymphatic tissues continuous from the obturator are dissected.

\section{Pararectal Space}

First, the ureter should be detached from the posterior leaf of broad ligament. The ureter is covered by the ligamentlike connective tissue continuous to the sacrouterine ligament, which is called "mesoureter." For the detachment of ureter, broad ligament is lifted up by two Kelly clamps, and then the ureter with the "mesoureter" is separated from the broad ligament using the scissors ( $\mathbf{F i g}$. 15A). Then, the operator should extend, using the index finger and scissors, this space

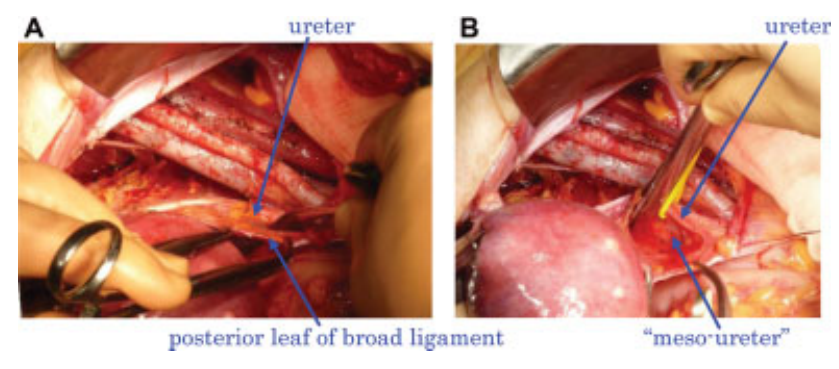

Fig. 15 Exploration of pararectal space (Latzko's space). First, the ureter with "mesoureter" should be detached from the posterior leaf of broad ligament using the scissors (A). Insertion of the finger or scissors toward the sacrouterine ligament results in the formation of pararectal space (Latzko's space) (B). Then, the ureter is isolated by the incision of "mesoureter," and is marked by a yellow tape.
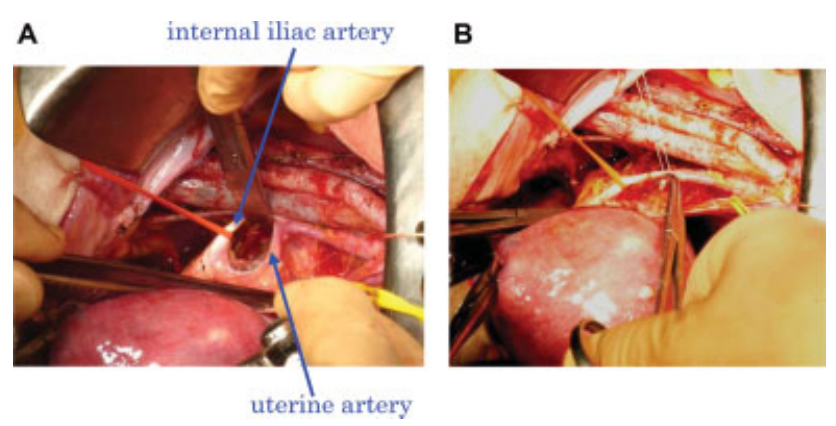

Fig. 16 Identification of uterine artery. Identification and isolation of the internal iliac artery disclose the presence of uterine artery, which runs with meandering fashion (A). This is clamped, cut, and ligated at the point of origin in the internal iliac artery (B). The superior vesical artery is usually preserved. Internal iliac artery is marked by a red tape.

between the ureter/"mesoureter" and the posterior leaf of broad ligament, anteriorly and posteriorly, and also extend caudally toward the uterine origin of sacrouterine ligament (-Fig. 15B). Here, the operator can touch the sacrouterine ligament by finger. This is an artificial space, but usually, there is little resistance from tissues during the procedure. This space extending downward along the posterior leaf of broad ligament is the pararectal space (Latzko's space). Then, the operator incises the "mesoureter," resulting in the complete isolation of ureter, which will be marked with a yellow-colored tape.

\section{Uterine Artery}

It is time to cut the uterine artery. The operator has already identified the internal iliac artery (lateral umbilical ligament), and can identify the uterine artery from its relatively cranial portion, by recognizing its characteristic feature of running with meandering fashion ( $\mathbf{- F i g}$. 16A). The uterine artery is clamped, cut, and ligated at the point of origin in the internal iliac artery ( - Fig. 16B). Here, the internal iliac artery is marked by red-colored tape. The superior vesical artery is recognized caudally from the uterine artery, and is preserved. Tracing the cut end of uterine artery downward, the operator finds the ureter running into the entrance of "ureter tunnel" under the uterine artery. Loose connective tissues surrounding the ureter and the uterine artery around the entrance of "ureter tunnel" are removed tentatively here, for easier access in the later surgery for vesicouterine ligament.

\section{Hypogastric Nerve}

For nerve-sparing radical hysterectomy, preservation of the hypogastric nerve is mandatory to maintain the urinary function of the patient. The posterior leaf of broad ligament is lifted again firmly by two Kelly clamps, and the whitecolored strings in the deep area are found. They are separated from the broad ligament by the scissors (-Fig. 17A). This is the hypogastric nerve, which will be marked with a whitecolored tape ( - Fig. 17B). If cancer invasion into the cardinal ligament or rectovaginal ligament is suspected, the hypogastric nerve cannot be preserved and will be sacrificed. 


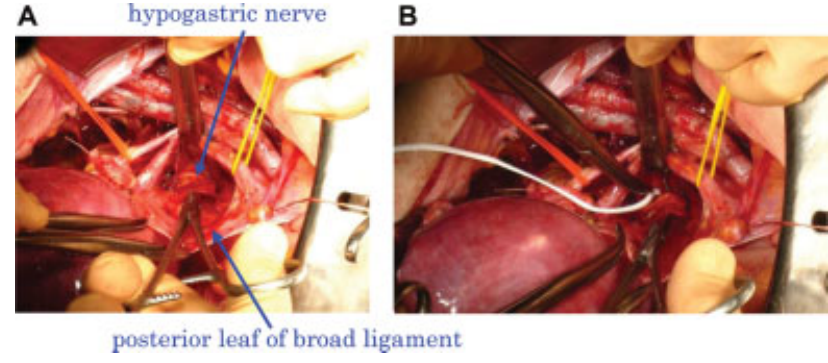

Fig. 17 Identification of hypogastric nerve. For nerve-sparing radical hysterectomy, preservation of the hypogastric nerve is mandatory. In the deep area of posterior leaf of broad ligament (A), white-colored strings are identified, detached, and marked using a white tape (B).

\section{Cardinal Ligament}

Now, the operator has reached the important point for the treatment of cardinal ligament, which exists between the paravesical space and the pararectal space (Latzko's space). Historically, this was the most dramatic scene of radical hysterectomy. First, the two long retractors are inserted into the two spaces, and pushed medially, keeping the retractors in parallel. Internal iliac artery is retracted laterally, and the ureter is pushed medially. Traction of the uterus upward is also necessary. Here, the operator recognizes the characteristic feature of cardinal ligament (-Fig. 18), which is based broadly on the pelvic wall and reaches the cervix, resembling that of Mount Fuji. However, clamping and cutting of ligament should not be done at this point, because dissection of lymphatic tissues of the cardinal ligament should precede.

Since the connective tissues having many small lymph nodes are still present within the cardinal ligament, the

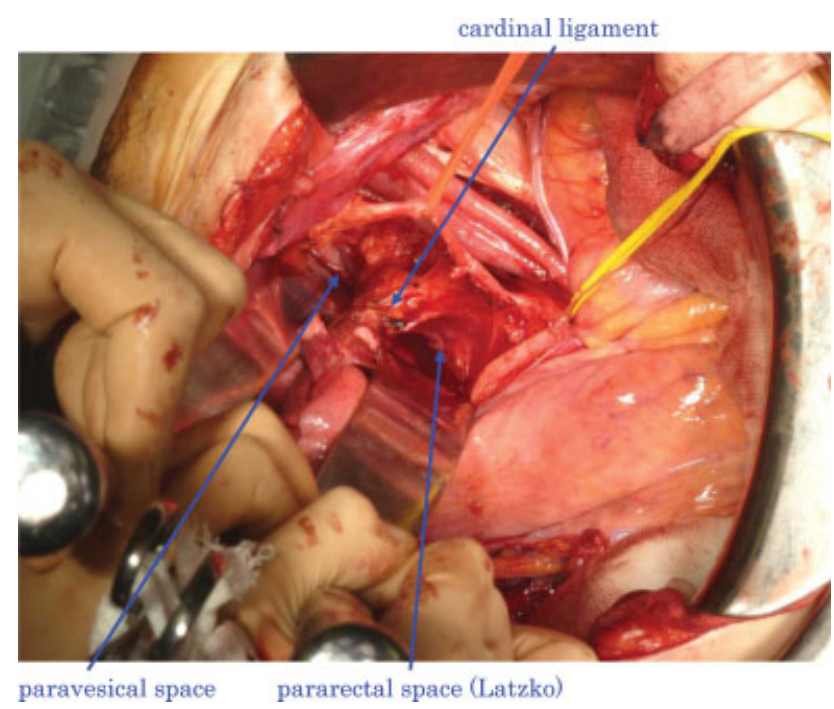

Fig. 18 Exploration of cardinal ligament. Two long retractors are inserted into the two spaces, i.e., one at paravesical space and another at pararectal space (Latzko's space). Internal iliac artery is retracted laterally (red tape), and the ureter is pushed medially (yellow tape). Between the two retractors, you can see clearly the cardinal ligament, which originates from the pelvic side wall and reaches the cervix. Clamping and cutting of ligament should not be done here, because dissection of lymphatic tissues of the cardinal ligament should precede.
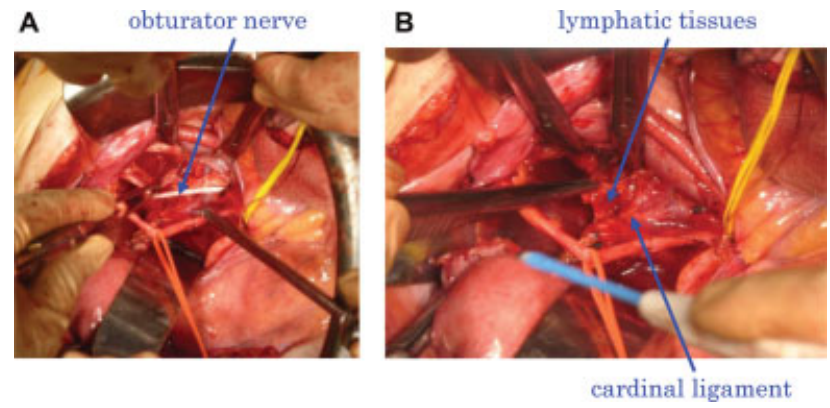

Fig. 19 Dissection of lymphatic tissues of the cardinal ligament. Removal of connective and lymphatic tissues in the area under the obturator nerve is carefully performed using aspirator and cautery (A). Dissection of lymph nodes in the cardinal ligament is very important to prevent the side wall recurrence (B).

operator should remove carefully these tissues existing in the area under the obturator nerve ( - Fig. 19A). This is an important procedure to prevent the side wall recurrence. Using the aspirator and monopolar or unipolar cautery, the operator carefully removes the lymphatic tissues from anterior and posterior sides of the cardinal ligament, and then the blood vessels appear gradually ( - Fig. 19B). The main trunk of the internal iliac vein also appears in the deep area of pelvic wall. Thus, such dissection expose the pelvic side wall as well as the arteries and veins in the cardinal ligament (-Fig. 20), including the deep uterine vein, which connects directly to the main trunk of the internal iliac vein. Cutline of the cardinal ligament has become truly clear after the dissection.

Now, the blood vessels of cardinal ligament are clearly visible, and therefore, their treatment can be done without the risk of massive bleeding. Thus, in modern radical hysterectomy, we do not encounter unexpected bleeding from the cardinal ligament. Usually, Kelly clamps are inserted into the avascular space ( - Fig. 21A), and first, the superficial vessels are clamped, cut, and ligated, either one by one or en bloc (-Fig. 21B). Then, the operator approaches the deep uterine vein, which is the

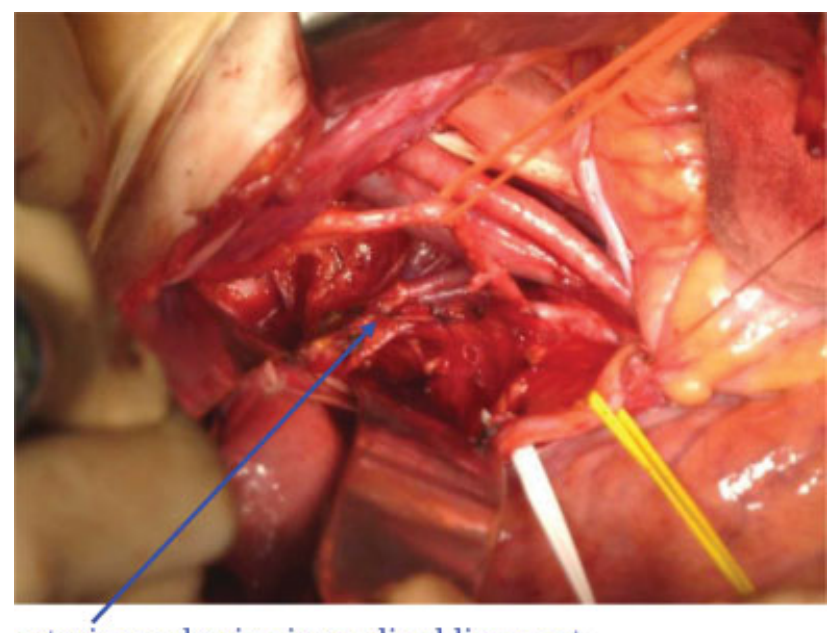

arteries and veins in cardinal ligament

Fig. 20 Appearance of the blood vessels of cardinal ligament. Dissection of lymphatic tissues discloses the arteries and veins in the cardinal ligament, pelvic side wall, and the main trunk of the internal iliac vein. Thus, the cutline of ligament has become clear. 

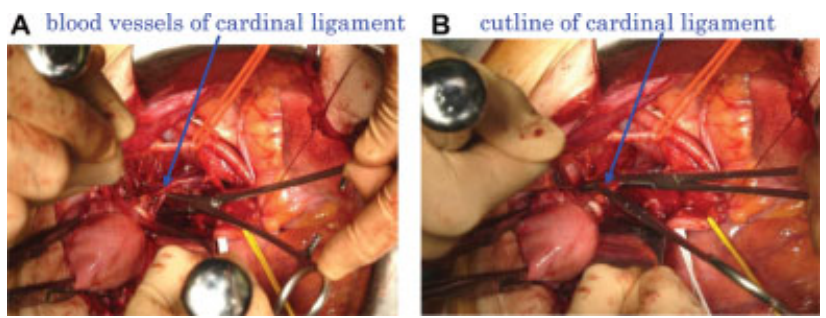

Fig. 21 Treatment of the superficial vessels of cardinal ligament. Since the blood vessels of cardinal ligament are clearly visible now, their treatment can be done without the risk of massive bleeding. Usually, Kelly clamps are inserted into the avascular space (A), and first, the superficial vessels are clamped, cut, and ligated, either one by one or en bloc (B).
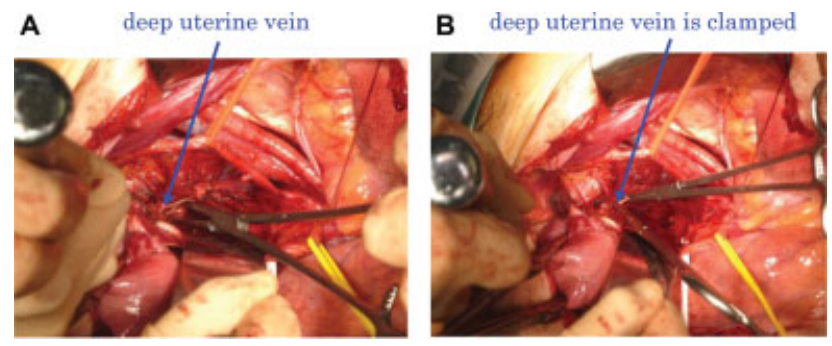

Fig. 22 Treatment of the deep uterine vein of cardinal ligament. In the deep area of the cardinal ligament, the deep uterine vein originating from the internal iliac vein is recognized $(A)$. This is the hallmark among the blood vessels of cardinal ligament, and is clamped (B), cut, ligated, and marked as the symbolic cut end of cardinal ligament.

hallmark of cardinal ligament (-Fig. 22A). In the deepest part of ligament, the deep uterine vein originating from the main trunk of internal iliac vein is recognized. And, it is clamped, cut, and ligated at the point close to the internal iliac vein ( - Fig. 22B). The treatment of cardinal ligament has been completed. Here, when the operator looks at the uterus and the entire image of anatomy, one can understand that the cut end of cardinal ligament (deep uterine vein) is running into the cervix, crossing just over the preserved hypogastric nerve.

The aforementioned surgical procedures, such as the exploration of paravesical space, pelvic lymphadenectomy, exploration of pararectal space, treatment of uterine artery, identification of hypogastric nerve, and the treatment of cardinal ligament, are also done in the same way for the left side.

\section{Tips and Warnings}

In each surgery for radical hysterectomy, the operator should always clarify the running of the external and internal iliac veins, the obturator nerve, and the iliopsoas muscle. Then, the operator becomes familiar with the anatomy of pelvic side wall, and soon recognizes that the running of internal iliac vein varies greatly, sometimes very close to pelvic wall and other times near the uterus. This is very important to decide the cutline of ligament for radical hysterectomy and also essential to assess how to perform "super-radical" hysterectomy, i.e., co-resection of the internal iliac vein if necessary.
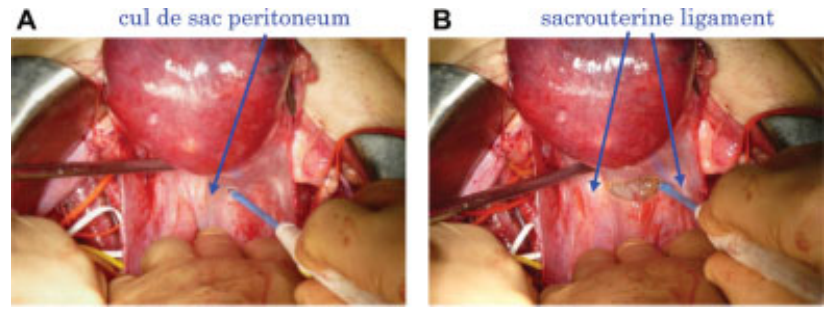

Fig. 23 Incision of cul-de-sac peritoneum. Now the operator starts the procedure of posterior part. The cul-de-sac peritoneum is stretched (A), and is incised using the scissors or monopolar cautery (B). Yellow-colored adipose tissues appear.

\section{Rectovaginal Ligament}

Then, the operator starts the posterior part of the surgical procedure, i.e., the treatment of rectovaginal ligament. The first step is to incise the peritoneum. The operator stretches the cul-de-sac peritoneum, and incise it using the scissors or monopolar cautery ( - Fig. 23A). Yellow-colored adipose tissues appear ( - Fig. 23B). Then, for separation of the rectum from the vagina, the operator inserts the midfinger into the incised space ( - Fig. 24A), sliding the finger just beneath the vaginal wall, not toward the rectal wall. Alternatively, the careful separation can be performed visually using the monopolar cautery. Then, the posterior leaf of broad ligament is incised downward, and the sacrouterine ligament is clamped, cut, and ligated.

The rectovaginal ligament is continuous from the sacrouterine ligament, very thick and firm, and directed downward to the sacrum. The operator should detach the rectum from the rectovaginal ligament medially, using the scissors ( - Fig. 24B). Then, using the index fingers, this space is extended downward, cranially, and caudally. Here, the pararectal space (Okabayashi) has been opened, and the rectum is completely free from the rectovaginal ligament and the vagina.

Then, the operator inserts one finger into the pararectal space (Okabayashi) and another into the pararectal space (Latzko's space), pushing the rectum medially and the hypogastric nerve laterally (-Fig. 25). This procedure is very important for prevention of rectal injury and for

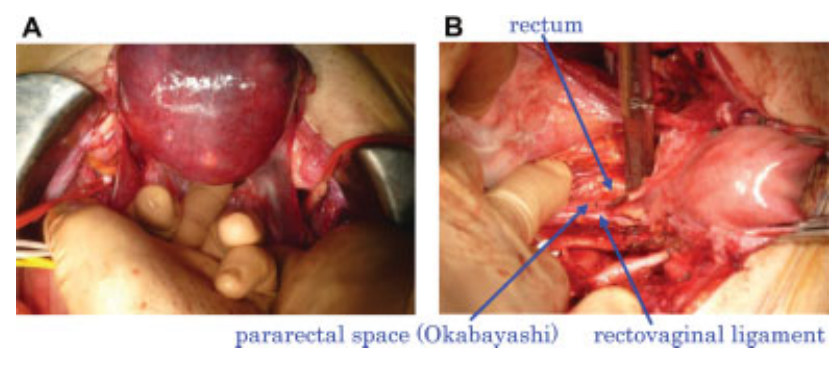

Fig. 24 Exploration of pararectal space (Okabayashi). The operator inserts the midfinger into the space between the vagina and the rectum, sliding the finger just beneath the vaginal wall (A). The posterior leaf of broad ligament is incised, and the sacrouterine ligament is clamped, cut, and ligated. Now, the rectovaginal ligament is recognized. The rectum is detached from the rectovaginal ligament using the scissors (B), and then the pararectal space (Okabayashi) has developed. 


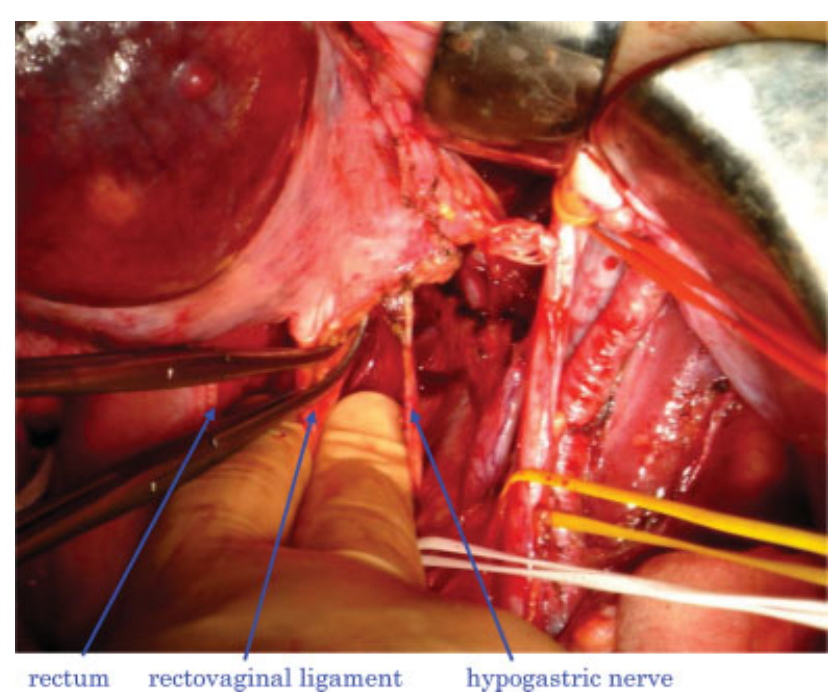

Fig. 25 Treatment of the rectovaginal ligament. The operator inserts one finger into the pararectal space (Okabayashi) and another into the pararectal space (Latzko's space), pushing the rectum medially and the hypogastric nerve laterally. The rectovaginal ligament between the two fingers is clamped, cut, and ligated. The procedure is repeated several times, until the cutline reaches to the cut end of cardinal ligament.

preservation of the hypogastric nerve. Thus, the rectovaginal ligament is now isolated from the surrounding tissues, and is clamped, cut, and ligated, several times until reaching the point where the cut end of cardinal ligament (deep uterine vein) is present. The height level of cutting depends on the degree of cancer invasion into the rectovaginal ligament.

This procedure is also performed for the left side. The cancer is now free from the posterior (rectum) and from the lateral (pelvic side wall) planes.

\section{Tips and Warnings}

Exploring the two different pararectal spaces, i.e., Latzko and Okabayashi, is also important in the surgeries for advanced ovarian cancer, in which the cancer cells metastasized to the cul-de-sac peritoneum. Not infrequently, the cul-de-sac peritoneum having the disseminated nodules can be separated from the rectum, and radical surgery is possible without resecting the rectum. In such case, exploration of the pararectal spaces makes it possible to dissect the peritoneum and to preserve the rectum safely.

\section{Vesicouterine Ligament}

Now, the operator starts the anterior part of the surgical procedure, i.e., the treatment of vesicouterine ligament. Of the two leaves of vesicouterine ligament, the anterior serves as the roof, while the posterior provides with the floor of ureter running.
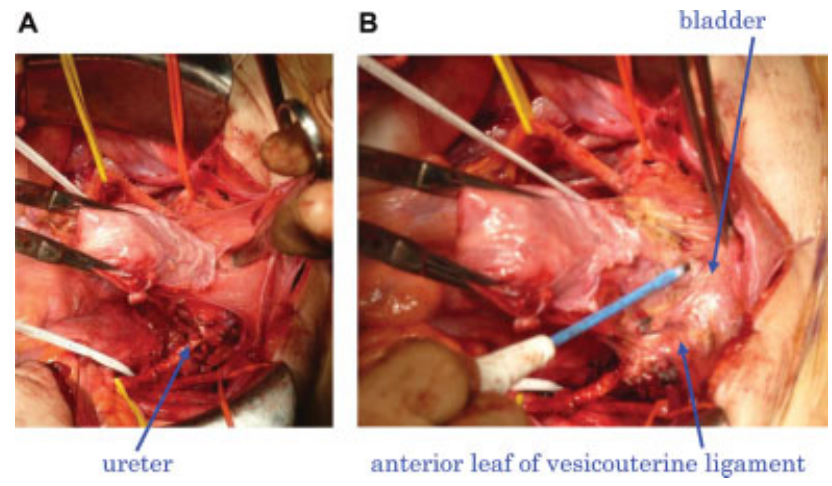

Fig. 26 Mobilization of bladder. The operator moves to anterior part. After incision of the connective tissues of vesicouterine fossa $(A)$, the bladder is detached from the cervix and mobilized downward using monopolar cautery (B). Loose connective tissues covering the anterior leaf of vesicouterine ligament are removed carefully. The dissection reaches to the level of $\sim 3 \mathrm{~cm}$ below the fornix.

\section{Anterior Leaf}

The first step is bladder mobilization at the midline of the cervix. The operator cut the loose connective tissue in the vesicouterine fossa with the scissors and monopolar cautery (-Fig. 26A). The surface of the cervical and vaginal walls gradually appears. If the invasion of cancer near the surface of cervix or vagina is suspected, the operator should not touch the cancer but treat only the connective tissues. Then, using the monopolar cautery, the operator removes carefully the superficial loose connective tissues in the lateral area, covering the vesicouterine ligament. The bladder is detached caudally to the level of about $3 \mathrm{~cm}$ below the fornix (-Fig. 26B). However, the depth level of dissection is dependent on the degree of vaginal invasion.

Since the anterior leaf of vesicouterine ligament has appeared at both sides, the operator understands the anatomical relationships among the ureter, vesicouterine ligament, and bladder (-Fig. 26B). The end point of the procedure is the complete removal of the anterior leaf that covers the ureter running into the bladder. This portion is named "ureter tunnel." The anterior leaf contains the cervicovesical vessels, and so, its injury will result in the massive bleeding. The following procedures should be performed step by step carefully.

First, the entrance of "ureter tunnel" should be identified. The assistants retract the uterus cranially, the ureter upward laterally, and the bladder caudally using retractors, and fix them. The cut end of uterine artery is lifted up to show clearly the entrance. Using the scissors, the operator can find the entrance of "ureter tunnel" at medial upper portion of the ureter. Then, the operator inserts the scissors into the tunnel ( - Fig. 27A), pushing the ureter laterally. Gradually, during the procedure, the inner wall of "tunnel" becomes clearly visible (-Fig. 27B).

The operator now understands the direction of "tunnel" by the gradual exploration of the "tunnel," and then some part of the anterior leaf of vesicouterine ligament is clamped ( - Fig. 28A), cut ( Fig. 28B), and ligated, being apart from the ureter. The operator can recognize the exit of "ureter tunnel," and so, for the remaining part of anterior leaf, Kelly clamps 
A cut end of uterine artery

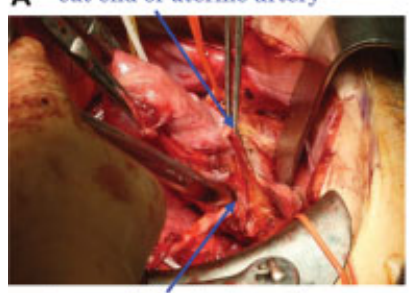

entrance of "ureter tunnel"
B

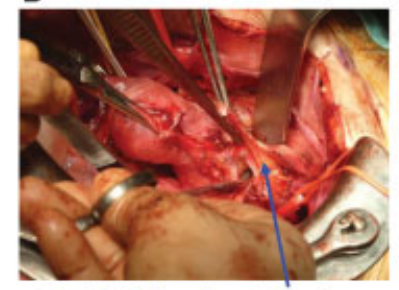

anterior leaf of vesicouterine ligament
Fig. 27 Approach to "ureter tunnel." The cut end of uterine artery is lifted and the ureter is mildly retracted cranially. The entrance of "ureter tunnel" appears just beneath the anterior leaf of vesicouterine ligament. Then, the operator inserts the scissors into the space between the ligament and the cervix (at 10 o'clock position for the right, and at 2 o'clock position for the left) (A), and pushes the scissors laterally (B).

A anterior leaf of vesicouterine ligament
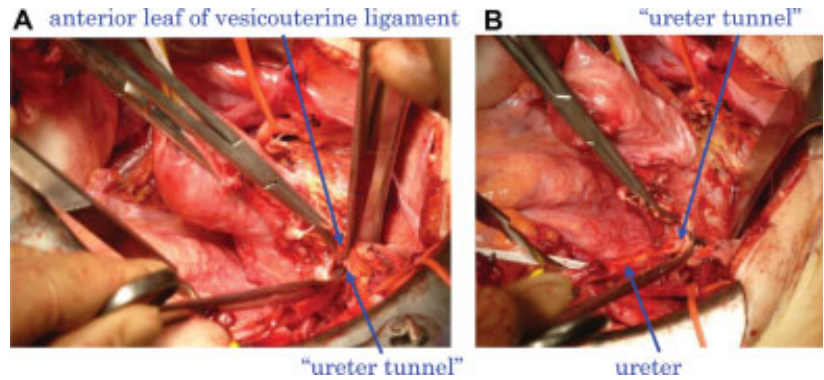

Fig. 28 Treatment of anterior leaf of vesicouterine ligament. When the ureter is pushed laterally using the scissors, some part of the anterior leaf of vesicouterine ligament is clamped (A), cut (B), and ligated.

are inserted ( - Fig. 29A). The remaining ligament is clamped, cut, and ligated. Thus, clamping and cutting of the anterior leaf are repeated usually two or three times, and result in the complete appearance of the ureter and its running into the bladder ( - Fig. 29B).

\section{Tips and Warnings}

For removal of the anterior leaf of vesicouterine ligament, it is important to identify the "ureter tunnel." For the right side, the entrance of "ureter tunnel" is present at the 10 o'clock position of the ureter ( 2 o'clock position for the left), i.e., upper and medial space between the ureter and ligament. When the operator insets the scissors into this point and push the ureter toward lateral and downward, the entrance of "ureter tunnel" will open. For safety, it is very important to watch the surface of ureter with the eyes of operator during the procedure.

\section{Posterior Leaf}

Then, the operator should detach the ureter from the floor and push the ureter laterally. Such lateral mobilization of the ureter is essential for the next step ( $\mathbf{F i g . ~ 3 0 A )}$ ). During this
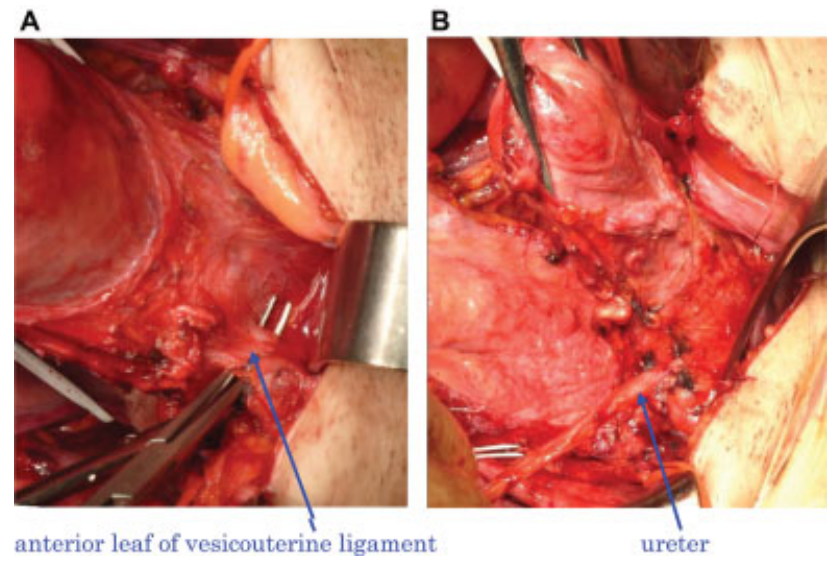

Fig. 29 Completion of anterior leaf of vesicouterine ligament. For the remaining part of anterior leaf of vesicouterine ligament, the ureter is pushed downward using the scissors, Kelly clamps are inserted (A), and the ligament is clamped, cut, and ligated. After completion of the anterior leaf, the ureter and its running into the bladder become clearly visible (B).
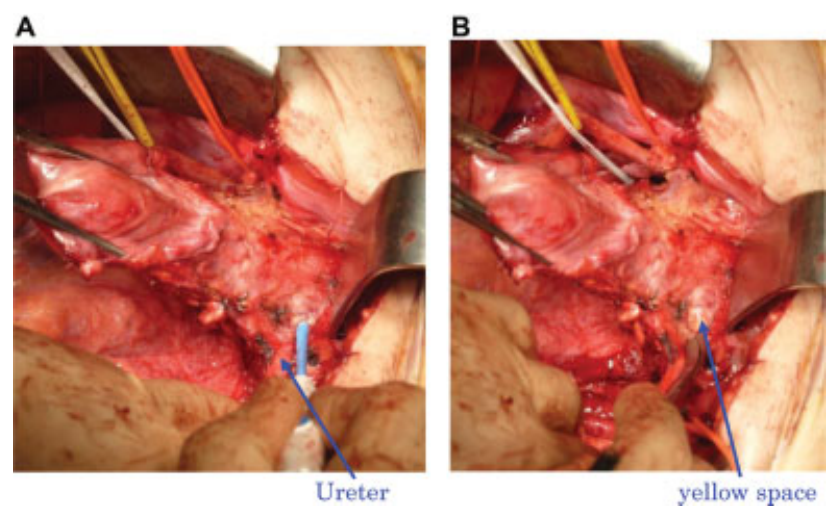

Fig. 30 Lateral mobilization of ureter. The ureter is mobilized completely by detachment from the posterior leaf of vesicouterine ligament using the monopolar cautery and scissors (A). During this procedure, the narrow space of yellow-colored adipose tissues appears between the paracolpium and the posterior leaf (B).

procedure, the narrow space of yellow-colored adipose tissues appears between the paracolpium and the posterior leaf ( - Fig. 30B). This yellow space is an important landmark for the next step. Thus, anatomical relationships among the ureter, the bladder, the posterior leaf of the vesicouterine ligament, and the paracolpium become clearly visible.

Now, the operator recognizes the posterior leaf of vesicouterine ligament running from the bladder to the cervix ( - Fig. 31A), where it meets with the cut end of the cardinal ligament. This is because the vesical vein in the posterior leaf anastomoses with the deep uterine vein. There is a space between the posterior leaf and the vagina, and its landmark is the yellow space. Thus, for actual identification and cutting of the posterior leaf, the operator should palpate the ligament, using the index finger and the thumb, and confirm the absence of vessels or bladder in the yellow space ( - Fig. 31B). The ligament contains two to three vesical veins anastomosing with the deep uterine vein, and their injury results in 


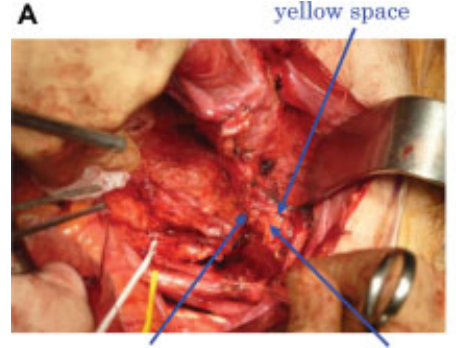

cut end of cardinal ligament posterior leaf of vesicouterine ligament

Fig. 31 Identification of posterior leaf of vesicouterine ligament. Now, the operator recognizes the posterior leaf of vesicouterine ligament running from the bladder to the cervix (A), where it meets with the cut end of cardinal ligament. This is because the vesical vein in the posterior leaf anastomoses with deep uterine vein. The operator should palpate the ligament, using the index finger and the thumb, and confirm the absence of vessels or bladder in the space between the paracolpium and the posterior leaf (B). You can see my index finger bulging at the yellow space.

massive bleeding. Therefore, palpation is very important to prevent the trauma to bladder or injury to vessels in the ligament.

\section{Tips and Warnings}

Where is the posterior leaf of vesicouterine ligament? Anatomically, the vesical vessels in the ligament flow from the bladder and continuous to deep uterine vein, and the ligament reaches to the cut end of cardinal ligament (deep uterine vein). To identify the medial border of the ligament, it is said that the yellow space can be seen between the posterior leaf and the paracolpium. Complete mobilization of the ureter laterally is needed to find it. Sometimes, it is not possible to find the yellow color visually. Therefore, it is important to confirm the space by palpation using the thumb and the index finger.

After palpation, the operator inserts vertically two Kelly clamps into the yellow space ( - Fig. 32A). Then, the posterior leaf of the vesicouterine ligament is clamped, cut, and ligated (-Fig. 32B).

The above-mentioned treatment for anterior and posterior leaves of the vesicouterine ligament is also performed for the left side.

\section{Paracolpium}

Now, the ligaments covering the ureter have completely been removed, and the paravaginal tissues (paracolpium) become evident ( - Fig. 33A). The operator will clamp, cut, and ligate it at the appropriate level ( - Fig. 33B).

This procedure is completed also for the left side.
A

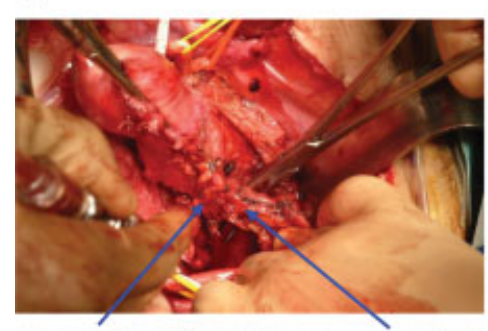

B cut ends of posterior leaf

cut end of cardinal ligament posterior leaf of vesicouterine ligament

Fig. 32 Treatment of posterior leaf of vesicouterine ligament. The Kelly clamps are inserted into the yellow space, which has been confirmed to be safe by palpation (A). The isolated posterior leaf of vesicouterine ligament is clamped, cut, and ligated (B).
A
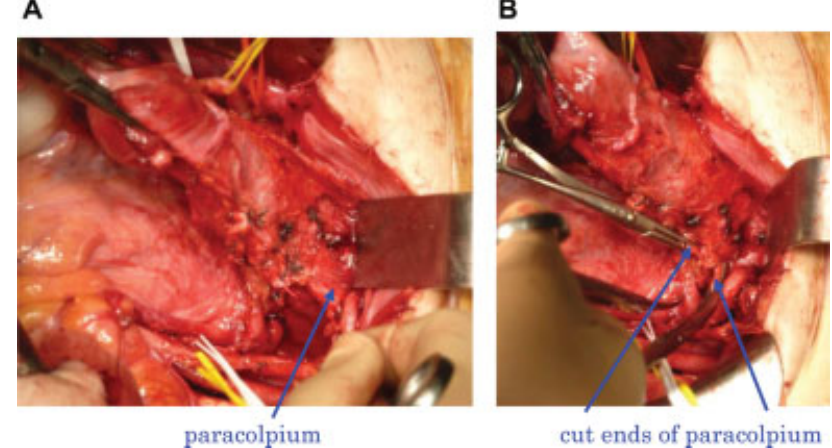

cut ends of paracolpium
Fig. 33 Treatment of paracolpium. After cutting the posterior leaf of vesicouterine ligament, the paracolpium appears $(A)$. At the appropriate level, the paracolpium is clamped, cut, and ligated (B).

\section{Amputation of Vagina}

Now the cancer is completely free from all of the ligaments supporting the uterus. Anterior vaginal wall is opened by the scalpel or monopolar cautery (-Fig. 34). The vagina is

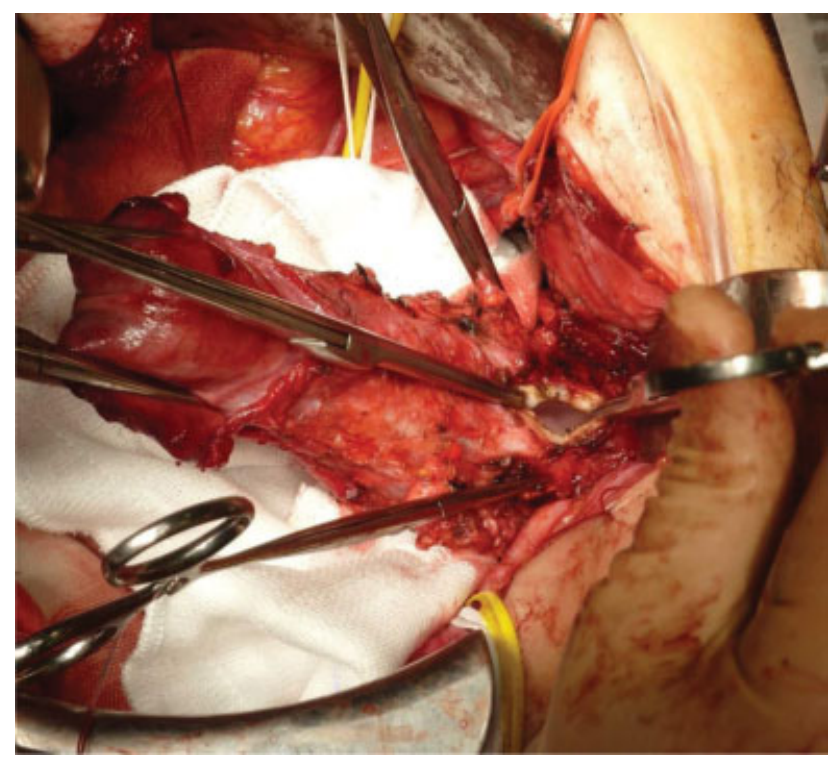

Fig. 34 Amputation of vagina. Anterior vaginal wall is opened by the scalpel or monopolar cautery. Vaginal vault is closed by suturing. Radical hysterectomy has been completed now. 
prepared by povidone-iodine, and a gauze is inserted into the vaginal cavity. The vaginal vault is closed with $Z$-figure sutures.

If the dissected pelvic lymph nodes are proven to be positive for metastasis, para-aortic lymphadenectomy till the level of inferior mesenteric artery will be added from here.

\section{Closing Abdomen}

The retroperitoneal space is washed by warm saline, and is confirmed for no bleedings and no foreign bodies. Two drains are placed in the field of lymphadenectomy. Pelvic peritoneum is not sutured in order to prevent formation of lymphocyst. The intestines are restored to normal position, and the abdomen is closed with each suture for the peritoneum, fascia, and skin.

\section{Conflict of Interest}

None declared.

\section{References}

1 Konishi I. Abdominal radical hysterectomy. Okabayashi method. In: Hiramatsu Y, Konishi I, Sakuragi N, Takeda S, eds. Mastering Basic Concepts and Essential Techniques OGS NOW, No. 5: Surgery for Cervical Cancer and Vulvar Cancer. Tokyo: Medical View; 2011:26-45

2 Wertheim $\mathrm{E}$. The extended abdominal operation for carcinoma uteri. Based on 500 operated cases. Am J Obstet Gynecol 1912; $66: 169-232$
3 Meigs JV. Carcinoma of the cervix: the Wertheim operation. Surg Obstet Gynecol 1944;78:195-199

4 Okabayashi H. Radical abdominal hysterectomy for cancer of the uterine cervix. Modification of the Takayama operation. Surg Obstet Gynecol 1921;33:335-341

5 Ogura T. Radical hysterectomy: Okabayashi original procedure. In: Kobayashi T, Nishimura T, Taki I, et al., eds. Cervical Cancer. Tokyo: Nakayama Shoten; 1970:129-151

6 Okabayashi H. Radical Hysterectomy for Cervical Cancer. Tokyo: Nippon Isho Shuppan; 1952

7 Kobayashi T. Abdominal Radical Hysterectomy with Pelvic Lymphadenectomy for Cancer of the Cervix. Tokyo: Nanzando; 1961

8 Fujii S, Takakura K, Matsumura N, et al. Anatomic identification and functional outcomes of the nerve sparing Okabayashi radical hysterectomy. Gynecol Oncol 2007;107(01):4-13

9 Mibayashi R. Mibayashi super-radical hysterectomy. In: Kobayashi T, Nishimura T, Taki I, et al., eds. Cervical Cancer. Tokyo: Nakayama Shoten; 1970:269-279

10 Mikami M, Ungár L, Matsuo K. Identification, technique, and outcome of super-radical hysterectomy for cervical cancer. In: Mikami M, ed. Surgery for Gynecologic Cancer. Tokyo: Springer: 2019:117-133

11 Ungár L, Pálfalvi L. Surgical treatment of lymph node metastases in stage IB cervical cancer: the laterally extended parametrectomy (LEP) procedure. Int J Gynecol Cancer 2003;13(05): 647-651

12 Höckel M. Laterally extended endopelvic resection. Novel surgical treatment of locally recurrent cervical carcinoma involving the pelvic side wall. Gynecol Oncol 2003;91(02):369-377 